\title{
HIGIENIZAÇÃO DE MÃOS: 20 ANOS DE DIVERGÊNCIAS ENTRE A PRÁTICA E O IDEALIZADO
}

\section{LAVADO DE MANOS: 20 AÑOS DE DIVERGENCIAS ENTRE LA PRÁCTICA Y LO IDEALIZADO}

\author{
HANDWASHING: 20 YEARS OF DIVERGENCES \\ BETWEEN PRACTICE AND THEORY
}

\author{
Elaine Drehmer de Almeida Cruz , Fabiana C Pimenta ${ }^{*}$, \\ Marinésia A P Palos ${ }^{* * *}$, Silvia Rita Marin DA SilVA ${ }^{* * *}$, ElUCIR GiR ${ }^{* * * *}$
}

\section{RESUMO}

\begin{abstract}
A higienização das mãos está associada à prevenção de agravos à saúde e tem sido objeto de pesquisas. Este estudo de revisão bibliográfica de 20 anos das publicações disponíveis na base de dados PubMed teve por objetivo caracterizar os relatos científicos sobre Higienização das Mãos e contextualizar divergências entre a prática e o ideal preconizado. Os resultados evidenciam a interferência do comportamento, do hábito, do ambiente e do contexto assistencial na baixa adesão a essa prática, bem como a necessidade de compreender e transformar a realidade, minimizando as divergências evidenciadas.
\end{abstract}

Palavras-chave: Higienização de mãos, adesão, comportamento, profissionais da saúde.

\begin{abstract}
Handwashing is related to the prevention of health hazards and has been a research issue. This retrospective, twenty-year literature review of publications -available at PubMed database- aimed to characterize scientific reports on handwashing (HW), and contextualize divergences between practice and theory. The results show behavior, habit and environment interference as well as care context explain the low adherence to this practice, besides the need to understand and transform reality, minimizing the evidenced divergences.
\end{abstract}

Key words: Handwashing, adherence, behavior, health professionals.

\section{RESUMEN}

La higienización de las manos está asociada a la prevención de daños a la salud y sigue siendo objeto de investigaciones. Este estudio de revisión bibliográfica de 20 años de las publicaciones disponibles en la base de datos PubMed tuvo por objetivo caracterizar los relatos científicos acerca de higienización de las manos y contextualizar divergencias entre la práctica y el ideal preconizado. Los resultados evidencian la interferencia del comportamiento, del hábito, del ambiente y del contexto asistencial en la pequeña adhesión a esa práctica, así como la necesidad de comprender y cambiar la realidad, minimizando las divergencias evidenciadas.

Palabras claves: Higienización de manos, adhesión, comportamiento, profesionales de la salud.

Fecha recepción: 29/03/07

Fecha aceptación: 19/03/09

\footnotetext{
* Enfermeira, docente junto ao Departamento de Enfermagem - Universidade Federal do Paraná, Paraná-Brasil, Email:elainedrehmer@yahoo.com.br

** Farmacêutica, docente junto ao Instituto de Patologia Tropical e Saúde Pública - Universidade Federal de Goiás - GoiâniaBrasil, Email:pimenta@iptsp.ufg.br

*** Enfermeira, docente junto à Faculdade de Enfermagem - Universidade Federal de Goiás Goiânia-Brasil, Email:marinesia. prado@terra.com.br

**** Enfermeira, docente junto à Escola de Enfermagem de Ribeirão Preto - Universidade de São Paulo - São Paulo-Brasil, Email:canini@eerp usp br

***** Enfermeira, docente junto à Escola de Enfermagem de Ribeirão Preto - Universidade de São Paulo - São Paulo-Brasil, Email:egir@eerp.usp.br
} 


\section{INTRODUÇÃO}

A assistência à saúde no ambiente hospitalar demanda aproximação física do profissional com os pacientes. As mãos são as estruturas corporais mais utilizadas no contato direto e se constituem no principal veículo de transmissão de microrganismos, representando o elo entre paciente, profissional e ambiente. A ruptura desse elo de transmissão exige a adoção de normas básicas de higiene no ambiente hospitalar, sendo a de maior impacto a higienização das mãos.

O termo "higienização de mãos" (HM) é genérico e se refere à ação de lavar as mãos com água e sabão comum, água e sabão com anti-séptico ou fricção com álcool a 70\%. O sabão proporciona a remoção mecânica da microbiota transitória da pele; quando associado a anti-séptico tem ação química letal aos microrganismos. O uso de álcool a $70 \%$ resulta em importante redução da carga microbiana transitória e residente, pela ação química e letal aos microrganismos(1).

Embora seja um ato simples e ensinado desde a infância como uma ação, principalmente de autocuidado, em serviços de saúde agrega produtos e técnicas que visam ampliar sua eficácia. Idealmente é recomendada cotidianamente antes e após o contato com o paciente e seu ambiente e apontada como fundamental para a segurança do paciente(2). Contudo, a adesão integral a essa prática vem sendo apontada como irreal e é evidenciada por estudos que apontam a baixa adesão dos profissionais de saúde à $H M$.

Frente ao exposto, este estudo tem os seguintes objetivos: caracterizar os relatos científicos sobre a temática "higienização das mãos" e contextualizar as divergências entre a prática e o ideal preconizado.

\section{MATERIAL E MÉTODO}

Esse estudo utilizou como método a revisão de literatura das publicações sobre o tema "higienização das mãos". Considerando os objetivos do estudo, inicialmente foram selecionados no Medical Subject Headings (MeSH) os seguintes descritores, os quais foram usados de forma associada: handwashing, compliance, behaviour. A base de dados utilizada para a busca on line dos estudos foi a PubMed (www. pubmed.gov) e os critérios de inclusão foram: artigos cujo tema central é Higienização das Mãos, publicados no período de 1987 a 2006 e inseridos na referida base de dados até janeiro de 2007. Foram identificadas 57 publicações, destas 37 foram incluídas de acordo com os critérios adotados neste estudo. A partir da leitura dos artigos, um formulário previamente construído foi preenchido para cada publicação com as seguintes informações: título, ano, país de publicação e país em que o estudo foi realizado, objetivos, delineamento do estudo, resultados, conclusões e recomendações.

\section{RESULTADOS E DISCUSSÃO}

Observou-se uma média de 1,85 publicações/ ano (p/a) sendo assim distribuídas: 1987 a 1991: 1 p/a; 1992 a 1996: 5 p/a; 1997 a 2001: 9 p/a; 2002 a 2006: 22 p/a; o aumento de p/a reflete uma tendência temporal progressiva para o estudo da temática. Houve predomínio norte-americano tanto na procedência dos periódicos (12 dentre 16 diferentes periódicos) quanto na realização do estudo (15 EUA, 6 Suíça, 3 Inglaterra, 2 Alemanha, 2 Austrália e 6 de outros diferentes países). Quanto ao tipo de pesquisa, estudos de revisão sistemática de literatura $(n=11 ; 29,7 \%)$ e observacionais $(\mathrm{n}=10 ; 27 \%)$ foram os mais freqüentes, seguidos de estudos de intervenção ( $n=9$; $24,3 \%)$, pesquisa de opinião/survey $(n=5$; $-13,5 \%)$, relato de caso $(n=1 ; 2,7 \%)$ e estudo caso-controle ( $\mathrm{n}=1 ; 2,7 \%)$.

A partir da caracterização dos resultados, conclusões e recomendações apresentados nos estudos, foi elaborada uma síntese em três categorias: Adesão à higienização de mãos: uma evidência em investigação; Baixa adesão à higienização de mãos: estratégias para a 
mudança; Buscando entender as divergências entre a prática e o ideal preconizado, as quais estão apresentadas a seguir.

\section{ADESÃO À HIGIENIZAÇÃO DE MÃOS: UMA EVIDÊNCIA EM INVESTIGAÇÃO}

Nesta categoria, as falhas na HM foram relacionadas à transmissão microbiana. As tentativas para mudar essa realidade abordaram o profissional de forma individual e os resultados foram limitados uma vez que o alvo da mudança não envolveu o contexto da assistência, mas o indivíduo(3). Embora haja entendimento de que a HM reduz o risco de infecção(4), a frequência da adesão dos profissionais foi inferior a 50\%(5-10). Ao serem indagados sobre o problema, os profissionais afirmaram ser possível aumentar a adesão à $\operatorname{HM}(11,12)$. Frente às oportunidades na prática assistencial, observou-se maior adesão dos profissionais à $\mathrm{HM}$ após executarem o cuidado e contato com fluidos corporais em detrimento à etapa antes do contato, revelando-se mais como uma prática de autocuidado que de cuidado ao paciente(13-15).

Pesquisas demonstraram que quanto maior a gravidade do paciente, mais frequentes foram as oportunidades para $\mathrm{HM} \mathrm{e}$ menor a adesão a essa prática(12), inclusive em pacientes hematológicos, os quais são mais suscetíveis às infecções(15). Nas investigações de surtos de infecção verificou-se estreita relação entre a sobrecarga de trabalho e falhas nas medidas de prevenção(16). Outro fator associado foi o uso de luvas que confere falsa segurança, predispondo a não troca entre exposições a diferentes sítios corporais de um mesmo paciente e a não HM após a sua retirada $(9,17)$.

Diferentes fatores foram apontados pelos profissionais como interferentes na adesão à HM: preferência para o uso de luvas, irritação cutânea, esquecimento, falta de tempo, estrutura física e insumos insuficientes(12). Revisão sistemática de literatura identifi- cou os seguintes elementos que interferem negativamente na adesão à HM: categoria profissional (médicos), o sexo (masculino), a atividade (trabalhar em unidade de cuidado intensivo e de alto risco para a contaminação grosseira), as oportunidades (alto número de oportunidades de HM por hora por paciente) e o uso de luvas(18).

Além da adesão à HM, outro fator relevante investigado foi a qualidade técnica do procedimento, havendo adequação em apenas $41,2 \%$ das práticas quando utilizado álcool (19). Consonante a esse resultado, o álcool tem sido recomendado por exigir menor tempo para a HM, ser prático ao uso, agir rapidamente e não lesar a pele de forma pronunciada $(16,20)$, resultando em adesão acima de $90 \%$ em situações que envolvem contato com material biológico(14).

\section{BAIXA ADESÃO À HIGIENIZAÇÃO DE MÃOS: ESTRATÉGIAS PARA A MUDANÇA}

Revisão sistemática da literatura evidenciou a relação entre o aumento da adesão à $\mathrm{HM} \mathrm{e}$ a redução nas taxas de infecção(7). Salientou a importância da eficácia das estratégias adotadas e discussão das variáveis que interferem nessa prática(21) priorizando-se ações que resultem na adesão e influenciem no comportamento, mais do que na elaboração de complexos estudos epidemiológicos(22). Sob a ótica dos profissionais, foram valorizadas as estratégias direcionadas a adequação da estrutura física, anti-sépticos menos irritantes e divulgação dos indicadores epidemiológicos de infecção hospitalar(10). Porém, outro estudo demonstrou que maior acessibilidade às pias não resultou em maior adesão à $\operatorname{HM}(23)$. Por outro lado, o uso de dispensadores automáticos de álcool contribuiu para a adesão quando comparado a dispensadores manuais(24), contrário aos achados de estudo anterior(6); outra questão apontada foi o risco de contaminação desses dispensadores(25). 
Quanto aos produtos, a introdução do uso de solução alcoólica sem a associação à estratégias que visem mudanças de comportamento foi apontada como pouco efetiva(26). Por outro lado, o feedback contínuo aos profissionais em relação à adesão à HM foi identificada como estratégia adequada $(21,27,28)$.

Ações educativas com uso de pôsteres, sinais luminosos, mensagens e envolvimento de pacientes foram ações utilizadas embora não haja estudos da sua efetividade em longo prazo(21), sendo valorizada a associação de diferentes estratégias(29).

A inclusão dos pacientes como parceiros de seu próprio cuidado foi utilizada como estratégia e resultou positivamente na adesão à $H M$ pelos profissionais $(30,31)$. Por outro lado, observou-se deficiências na prática de HM realizada pelos pacientes, após o uso do banheiro e da comadre(32).

Programas educacionais devem valorizar a HM e seus aspectos epidemiológicos, além dos comportamentais, motivacionais e envolvimento dos profissionais e líderes $(16,18,33)$ uma vez que o comportamento dos pares influencia o grupo $(6,23)$. A vivência de situações críticas pode contribuir para maior adesão; ao comparar a adesão de estudantes de medicina à HM, antes e após o surto de SARS (Severe Acute Respiratory Syndrome) pesquisadores demonstraram o importante impacto deste evento na motivação para a $\mathrm{HM}(34)$. Essa prática deve ser construída durante o ensino como um valor profissional(35).

A adoção de estratégias educativas associadas ao envolvimento dos profissionais, mesmo frente a altas taxas de infecção, resultou em maior adesão à $\operatorname{HM(36).~As~estratégias~}$ devem considerar os fatores associados a não a adesão de acordo com os níveis envolvidos, individual, grupal e institucional $(3,18)$, reconhecendo que adesão e crenças são variáveis que necessitam abordagem diversificada e multidisciplinar(10).

\section{BUSCANDO ENTENDER AS DIVERGÊNCIAS ENTRE A PRÁTICA E O IDEAL PRECONIZADO}

A compreensão do comportamento humano tem sido valorizada mais recentemente como fundamental no contexto da HM. Conhecimento, motivação, intenção, percepção, expectativas e pressão social são algumas das variáveis cognitivas utilizadas em modelos teóricos para explicar o comportamento humano(37).Kretzer e Larson em 1998 revisaram teorias comportamentais e sua aplicabilidade a partir da percepção de riscos, atitudes, expectativas, opinião e normas subjetivas, intenção e estágios do processo de mudança dos profissionais(3). Concluíram ser importante a associação de estratégias que considerem elementos do comportamento humano e do contexto organizacional para atingir mudanças significativas e duradouras na prática ideal da $\operatorname{HM}(3)$.

Embora as técnicas envolvidas nessa prática sejam simples, sua interdependência com as ciências do comportamento as torna complexas(38) e dependentes de um conjunto de fatores como atitudes, crenças e conhecimento(39). Sob esta ótica, atitude positiva, rigoroso controle de comportamento e percepção do valor atribuído pelos superiores à HM foram associados com a intencionalidade da adesão(12). Por outro lado, demonstraram que o hábito pode suplantar a intenção de realizar determinada ação. Neste sentido, para os pesquisadores embora os profissionais valorizem e reconheçam a importância da HM, o hábito inadequado resulta em não adesão(12).

\section{CONSIDERAÇÕES FINAIS}

Ao caracterizarmos as publicações sobre a "higienização das mãos" e contextualizarmos as divergências evidenciadas entre a prática 
e o ideal preconizado, concluímos não ser uma problemática de fácil e única solução. Enquanto profissionais da área da saúde, reconhecemos que práticas inadequadas em virtude do comportamento humano fazem parte do cotidiano e acrescentam riscos aos trabalhadores e aos pacientes. Portanto, necessitamos entender os determinantes da baixa adesão à HM de acordo com a realidade institucional e explorar alternativas facilitadoras para mudanças.

Muitas lacunas ainda necessitam ser exploradas por meio de pesquisas delineadas a partir da prática. Seus resultados devem ser revertidos no fazer, transformando a realidade e minimizando as divergências entre a prática e o idealizado, proporcionando maior segurança e qualidade na assistência em saúde.

\section{REFERÊNCIAS}

1. Boyce JM, Pittet D. Guideline for hand hygiene in health-care settings: recommendations of the Healthcare Infection Control Practices Advisory Committee and HICPAC/SHEA/APIC/IDSA Hand Hygiene Task Force. MMWR Morb Mortal Wkly Rep. 2002;51:1-45.

2. Anvisa. Agência Nacional de Vigilância Sanitária. Segurança do paciente. Higienização das mãos. Brasília (Brasil): Ministério da Saúde, 2008.

3. Kretzer, EK, Larson EL. Behavioral interventions to improve infection control practices. AJIC, 1998;26(3):245-53.

4. Nobile CGA, Montuori P, Diaco E, Villari P. Healthcare personnel and decontamination in intensive care units: knowledge, attitudes, and behavior in Italy. J Hosp Infect. 2002;51: 226-32.

5. Meengs MR, Giles BK, Chisholm CD, Cordell WH, Nelson DR. Handwashing frequency in an emergency department. J Emerg Nurs. 1995;20(3):183-8.

6. Muto CA, Sistrom MG, Farr BM. Hand hygiene rates unaffected by installation of dispensers of a rapidly acting hand antiseptic. AJIC. 2000;28(3):273-6.

7. Pittet D. Improving adherence to hand hygiene practice: a multidisciplinary approach. Emerg Infect Dis. 2001;7(2):23440.

8. Lipsett PA, Swoboda SM. Handwashing compliance depends on professional status. Surg Infect. 2001;2(3):241-5.

9. Kim PW, Roghmann M, Perencevich EN, Harris AD. Rates of hand disinfection associated with glove use, patient isolation, and changes between exposure to various body sites. AJIC. 2002;31(2):97-103.

10.Patarakul K, Tan-Khum A, Kanha S, Padungpean D, Jaichaiyam OO. Cross-sectional survey of hand-hygiene compliance and attitudes of health care workers ans visitors in the intensive care units at King Chulalongkorn Memorial Hospital. J Med Assoc Thai. 2005; 88 Suppl 4:S287-S293.

11.Camins BC, Fraser VJ. Reducing the risk of health care-associated infections by complying with CDC hand hygiene guidelines. Jt Comm J Qual Patient Saf. 2005;31(3):173-9.

12.Pessoa-Silva CL, Posfay-Barbe MD, Touveneau S, Perneger TV, Pittet D. Attitudes and perceptions toward hand hygiene among healthcare workers caring for critically ill neonates. ICHE. 2005;26(3):305-11.

13.Pittet D. Compliance with hand disinfection and its impact on hospital-acquired infections. J Hosp Infect. 2001;48 Suppl A:S40-6.

14. Wendt C, Knautz D, Baum H. Differences in hand hygiene behavior related to the contamination risk of healthcare activities in different groups of healthcare workers. ICHE. 2004;25(3):203-6.

15.Saba R, Inan D, Seyman D, Gul G, Senol YY, Turhan O, Mamikoglu L. Hand hygiene compliance in a hematology unit. Acta Haemat. 2005;113(3):190-3.

16.Pittet D. Hand hygiene: improved standards and practice for hospital care. Cur Opin Infect Dis. 2003;16(4):327-35.

17.Girou E, Chai SHT, Oppein F, Legrand P, Ducellier D, Cizeau F, Brun-Buisson C. 
Misuse of gloves: the foundation for poor compliance with hand hygiene and potential for microbial transmission? J Hosp Infect. 2004;57:162-9.

18.Pittet D. Improving compliance with hand hygiene in hospitals. ICHE. 2000;21(6):381-6.

19.Tvedt C, Bukholm G. Alcohol-based hand disinfection: a more robust hand-hygiene method in an intensive care unit. J Hosp Infect. 2005;59:229-34.

20.Kramer A, Berning T, Kampf G. Clinical double-blind trial on the dermal tolerance and user acceptability of six alcohol-based hand disinfectants for hygienic hand disinfection. J Hosp Infect. 2002;51:114-20.

21.Nakaioba S, Hayward A. The effectiveness of intervention aimed at increasing handwashing in healthcare workers- a systematic review. J Hosp Infect. 2001;47:173-83.

22.Nystrom, B. Impact of handwashing on mortality in intensive care: examination of the evidence. ICHE. 1994;15(7):435-6.

23.Lankford MG, Zembower TR, Trick WE, Hacek DM, Noskin GA, Peterson LR. Influence of role models and hospital design on hand hygiene of health care workers. Emerg Infect Dis. 2003;9(2):217-23.

24.Larson EL, Albrecht S, O'keefe M. Hand hygiene behavior in a pediatric department and a pediatric intensive care unit: comparison of use of 2 dispenser system. Am J Crit Care. 2005;14(4),304-311.

25.Wurtz R, Moye G, Jovanovic B. Handwashing machines, handwashing compliance, and potential for cross-contamination. AJIC. 1994;22(4):228-30.

26. Whitby M, Mclaws ML, Ross MW. Why healthcare workers don't wash their hands: a behavioral explanation. ICHE. 2006;27(5):484-92.

27.Dubbert PM, Dolce J, Richter W, Miller M, Chapman SW. Increasing ICU staff handwashing: effects of education and group feedback. ICHE. 1990;11(4):191-3.

28.Salemi C, Canola MT, Eck EK. Handwashing and physicians: how to get them together. ICHE. 2002;23(1):32-5.
29.Dorsey ST, Cydulka RK, Emerman CL. Is handwashing teachable?: Failure to improve handwashing behaviour in an urban emergency department. Acad Emerg Med. 1996;3 (4):360-5.

30.McGuckin M, Taylor A, Martin V, Porten L, Salcido R. Evaluation of a patient educational model for increasing hand hygiene compliance in an inpatient rehabilitation unit. AJIC. 2004;32:235-8.

31.McGuckin M, Waterman R, Porten L, Bello S, Caruso M, Juzaitis B, Krug E, Mazer S, Ostrawski S. Patient education model for increasing handwashing compliance. AJIC. 1999;27(4):309-14.

32. Ward D. Improving patient hand hygiene. Nurs Stand. 2003;17(35):39-42.

33.Larson E, Kretzer EK. Compliance with handwashing and barrier precautions. J Hosp Infect. 1995;30 Suppl:88-106.

34.Wong TW, Tam WW. Handwashing practice and the use of personal protective equipment among medical students after the SARS epidemic in Hong Kong. AJIC. 2005;33:580-6.

35. Feather A, Stone SP, Wessier A, Bousicot KA, Pratt C. Now please wash your hands: the washing beaviour of final MBBS candidates. J Hosp Infect. 2000;45(1):62-4.

36.Johnson PDR, Martin R, Burrell LJ, Grabsch EA, Kirsa SW, O’keeffe J, Mayhall BC, Edmons D, Barr W, Bolger C, Naidoo $\mathrm{H}$, Grayson ML. Efficacy of an alcohol/ chlorexidine hand hygiene program in a hospital with high rates of nosocomial methicilin-resistant Staphylococcus aureus (MRSA) infection. Med J Austr. 2005; 183(10):509-14.

37.Pittet D. The lowbury lecture:behaviour in infection control. J Hosp Infect. 2004;58:113.

38.Jumaa PA. Hand hygiene: simple and complex. Intern Journal Infec Dis. 2005;9(1):314.

39.Creedon SA. Healthcare workers' hand decontamination practices: compliance with recommended guidelines. J Adv Nurs. 2005;51(3):208-16. 